\title{
ORIGINAL ARTICLE \\ Maternal high-fat diet impacts endothelial function in nonhuman primate offspring
}

\author{
L Fan $^{1,2}$, SR Lindsley ${ }^{1}$, SM Comstock ${ }^{1}$, DL Takahashi ${ }^{1}, \mathrm{AE}$ Evans ${ }^{1}, \mathrm{G}-\mathrm{W} \mathrm{He}^{2}, \mathrm{KL}$ Thornburg ${ }^{3}$ and $\mathrm{KL} \mathrm{Grove}^{1}$
}

OBJECTIVE: The link between maternal under-nutrition and cardiovascular disease (CVD) in the offspring later in life is well recognized, but the impact of maternal over-nutrition on the offspring's cardiovascular function and subsequent risk for CVD later in life remains unclear. Here, we investigated the impact of maternal exposure to a high-fat/calorie diet (HFD) during pregnancy and early postnatal period on endothelial function of the offspring in a nonhuman primate model.

METHODS: Offspring, naturally born to either a control (CTR) diet (14\% fat calories) or a HFD (36\% fat calories) consumption dam, were breast-fed until weaning at about 8 months of age. After weaning, the offspring were either maintained on the same diet (CTR/CTR, HFD/HFD), or underwent a diet switch (CTR/HFD, HFD/CTR). Blood samples and arterial tissues were collected at necropsy when the animals were about 13 months of age.

RESULTS: HFD/HFD juveniles displayed an increased plasma insulin level and glucose-stimulated insulin secretion in comparison with CTR/CTR. In abdominal aorta, but not the renal artery, acetylcholine-induced vasorelaxation was decreased remarkably for HFD/HFD juveniles compared with CTR/CTR. HFD/HFD animals also showed a thicker intima wall and an abnormal vascular-morphology, concurrent with elevated expression levels of several markers related to vascular inflammation and fibrinolytic function. Diet-switching animals (HFD/CTR and CTR/HFD) displayed modest damage on the abdominal vessel. CONCLUSION: Our data indicate that maternal HFD exposure impairs offspring's endothelial function. Both early programming events and postweaning diet contribute to the abnormalities that could be reversed partially by diet intervention.

International Journal of Obesity (2013) 37, 254-262; doi:10.1038/ijo.2012.42; published online 27 March 2012

Keywords: over-nutrition; programming; intrauterine; endothelium; juvenile offspring

\section{INTRODUCTION}

The worldwide prevalence of childhood obesity has increased dramatically in the past several decades. ${ }^{1}$ Numerous epidemiological studies have documented a close association between early-onset obesity and the risk for developing cardiovascular diseases (CVDs) in adulthood. ${ }^{2-4}$ Maternal health and diet during pregnancy and the early postnatal period is now known to be the major contributing factor to the manifestation of metabolic disorders in obese children. ${ }^{5-7}$ A clear association has been established in humans between nutritional restriction and placental insufficiency during pregnancy with the connection to CVD in their offspring later in life. These effects have been confirmed in sheep models. ${ }^{8-12}$ In addition, there are data in rodent models, ${ }^{13-15}$ demonstrating that maternal over-nutrition can impair cardiovascular function in obese offspring as adults; however, it was not determined if the endothelial dysfunction occurred before the onset of obesity. Our current study aims to determine the relative impact of exposure to a high-fat/calorie diet (HFD) during the prenatal and/or postnatal period on endothelial function in juvenile nonhuman primate (NHP) offspring.

We have established a maternal HFD NHP model, ${ }^{16}$ where monkeys are maintained on a HFD before and throughout pregnancy and lactation. Using this model, our ongoing studies $^{16-21}$ demonstrated that consumption of a HFD during pregnancy leads to broad developmental health issues in the offspring, independent of maternal obesity and insulin resistance. These studies have found that the fetal offspring demonstrated restricted fetal growth, placental insufficiency accompanied by a decrease in placental blood flow volume, increased release of cytokines, dyslipidemia and an increased deposition of fat in the liver. Moreover, after birth, the HFD offspring displayed catch-up growth, increased fat mass and persistent fatty liver. These are all hallmarks for an increased risk of CVD. However, few studies to date have been conducted on the effects of maternal HFD intake on the cardiovascular system of the offspring, especially on an NHP model.

High-fat dietary intake is a major risk factor for the development of obesity. Obesity is closely associated with a number of established cardiovascular risk factors, including diabetes mellitus, insulin resistance, dyslipidemia and hypertension, which are cumulatively damaging to endothelial function. ${ }^{22-24}$ The structural and functional integrity of the vascular endothelium has a crucial role in cardiovascular homeostasis. Abnormal endothelial function is a well-recognized precursor of atherosclerotic disease. Under normal circumstances, the vascular endothelium releases various vasodilator and vasoconstrictor substances that regulate local vascular tone to ensure adequate blood flow, as well as regulate platelet aggregation and leukocyte adhesion to the endothelium. All of these effectively counteract the onset of atherosclerosis. ${ }^{25}$ On the contrary, in a state of insulin resistance, such as obesity and diabetes, endothelium-dependent vasodilator

${ }^{1}$ Division of Neuroscience, Oregon National Primate Research Center, Oregon Health and Science University, Beaverton, OR, USA; ${ }^{2}$ Cardiovascular Research Laboratory, Starr Academic Center, Providence Heart and Vascular Institute, Portland, OR, USA and ${ }^{3}$ Heart Research Center, Oregon Health and Science University, Portland, OR, USA. Correspondence: Dr KL Grove, Division of Neuroscience, Oregon National Primate Research Center, Oregon Health and Science University, 505 NW 185th Avenue, Beaverton, OR 97006, USA. 
response, the most useful measurement of endothelium function, ${ }^{26}$ is depressed. ${ }^{27-30}$

Based on the evidence that endothelial dysfunction is the central pathogenic feature of metabolic-disease-associated cardiovascular disorders, ${ }^{30-32}$ and based on our previous findings, we hypothesized that maternal HFD consumption during pregnancy and the perinatal period would impair the endothelial function of the offspring. In order to test our hypothesis, we collected abdominal aorta and renal arteries from 13-month-old NHP juvenile animals to test endothelial function, using multiple techniques. Apart from understanding the programming effects on the CVD risk in the juvenile offspring as a consequence of maternal obesity, this study also aimed to determine the contribution of dietary vs early programming events to endothelial dysfunction. In addition, these studies investigate the possible beneficial contribution of dietary intervention in juvenile animals, after the potential early programming events have occurred.

\section{MATERIALS AND METHODS}

NHP model

All animal procedures were in accordance with the guidelines of the Institutional Animal Care and Use Committee of the ONPRC (Oregon National Primate Research Center) and conformed to National Institutes of Health guidelines on the ethical use of animals. The complete characterization of the maternal and fetal phenotype has been described in our previous studies. ${ }^{22-27}$

\section{Adults}

Young adult female Japanese macaques (Macaca fuscata) were age(5-7 years old) and weight-matched $(7-9 \mathrm{~kg})$ and maintained on a control (CTR) or a HFD up to 5 years. The CTR diet contained $14 \%$ calories from fat (Lab Diet, 5052, Purina Mills Inc., St Louis, MO, USA) supplemented with fruits and vegetables, and the HFD supplied $36 \%$ of calories from fat (Test Diet, 5LOP, Purina Mills Inc.) supplemented with calorically dense treats. The composition of this HFD represents a typical Western diet in regards to the saturated fat content. All animals were socially housed in indoor/outdoor pens (male-to-female ratio of 1-2:5-9) and had ad libitum access to food and water.

\section{3-Month-old juveniles}

Offspring, naturally born to either a CTR or a HFD consumption dam, were maintained with their dams until about 8 months of age ( $249 \pm 4$ days). After weaning, some offspring were maintained on the same diet as their moms, fed either a CTR diet (group 1: CTR/CTR, denoted as C/C) or a HFD (group 2: $\mathrm{HFD} / \mathrm{HFD}$, denoted as $\mathrm{H} / \mathrm{H}$ ); others underwent a diet switch from either HFD to CTR (group 3: HFD/CTR, denoted as H/C) or from CTR to HFD (group 4: CTR/HFD, denoted as $\mathrm{C} / \mathrm{H}$ ). All the juveniles were socially housed. At $\sim 13$ months of age (393 \pm 4 days), all juveniles from the four treatment groups were sent to necropsy for blood and tissue collection. The data collected from these animals were from three different cohorts of animals over 3 years and were balanced for offspring sex and maternal diet sensitivity.

\section{Measurements of metabolic phenotypes}

Before necropsy, all the juvenile offspring underwent a morning fast. In the afternoon, the animals were deeply anesthetized with Nembutal and the body weights were measured. Blood samples were collected for the assessment of their metabolic status, including blood glucose level (determined by One-Touch Glucometer (LifeScan, Inc., Milpitas, CA, USA), which demonstrated a good analytical performance with a bias $<10 \%$ from the comparative clinic and laboratory methods in humans ${ }^{33}$ ), plasma insulin and triglycerides levels, and serum free fatty acids level (assayed by using the corresponding kit). Homeostatic model assessment of insulin resistance (HOMA-IR) was calculated by the formula glucose $\left(\mathrm{mg} \mathrm{dl}^{-1}\right) \times$ insulin $\left(\mu \mathrm{Uml}^{-1}\right) / 405$. Area under the curve for glucose (GAUC) and insulin secretion (IAUC) were calculated based on an ivGT test (intravenous glucose tolerance test), which was conducted for each animal 1 week before it was sent to necropsy. In detail, after an overnight fast, the animal was then sedated with Telazol $\left(3 \mathrm{mg} \mathrm{kg}^{-1}\right)$ and a baseline blood sample was collected. After an intravenous administration of a high dose of glucose $\left(0.6 \mathrm{~g} \mathrm{~kg}^{-1}\right.$ of sterile $50 \%$ dextrose solution), a series of blood samples were collected at $1,3,5,10,20,30,45$ and 60 min thereafter. GAUC and IAUC were then calculated based on the glucose and insulin concentrations at each time point. In addition, total body fat for each animal was determined concurrently with the ivGTT test by conducting DEXA scans (Hologic Discovery A, Hologic Inc., Bedford, MA, USA) during the sedation period.

\section{Preparation of abdominal aorta and renal artery tissue}

At necropsy, the far end of the descending abdominal aorta rings and the renal arteries were removed and dissected, with some of the tissue being snap frozen, and some submersed in $4 \%$ paraformaldehyde $(\mathrm{pH}=7.4)$ or neutral formalin.

Organ bath technique and the recording of acetylcholine-induced vasorelaxation

Freshly collected abdominal and renal arterial rings, in 3-mm lengths, were mounted in an organ bath, followed by a 60 -min resting period. ${ }^{34,35}$ The rings were then stretched in progressive steps to reach their optimal lumen pressure. After equilibration for another $60 \mathrm{~min}$, the rings were pre-contracted by $\mathrm{U}_{46619}$ (9,11-dideoxy-11 $\alpha, 9 \alpha$-epoxy-methanoprostaglandin $\mathrm{F}_{2 \alpha i}$ Cayman Chemical Company, Philadelphia, PA, USA; $10 \mathrm{~nm}$ ). When the contraction reached a stable plateau, cumulative doses of acetylcholine (ACh; Sigma Chemical Co., St Louis, MO, USA; $-10 \sim-5.5$ LogM) were added by 0.5 -Log steps to produce a vasorelaxant response. The cumulative concentration-relaxation curves were then recorded. The vasorelaxant response to $\mathrm{ACh}$ at each dose point for each ring was finally expressed as a percentage decrease from $\mathrm{U}_{46619}$-induced pre-contraction. $\mathrm{EC}_{50}$ value for each curve was also calculated.

Kreb's solution of the following composition (mM): $\mathrm{Na}^{+} 144, \mathrm{~K}^{+}$5.9, $\mathrm{Ca}^{2+} 2.5, \mathrm{Mg}^{2+} 1.2, \mathrm{Cl}^{-} 128.7, \mathrm{HCO}_{3}^{-} 25, \mathrm{SO}_{4}^{2-} 1.2, \mathrm{H}_{2} \mathrm{PO}_{4}^{-}$1.2, Glucose 11, aerated with a gas mixture of $95 \% \mathrm{O}_{2}$ and $5 \% \mathrm{CO}_{2}$ at $37 \pm 0.1{ }^{\circ} \mathrm{C}$ was utilized throughout the experiments.

Quantitative real-time polymerase chain reaction and gene expression level determination

The total RNA of the arterial tissue was isolated. Then, $1 \mu \mathrm{g}$ RNA of each sample was prepared for real-time reaction and the mRNA expression level for each gene was measured by quantitative real-time polymerase chain reaction using a PRISM 7700 Sequences Detector System (Applied Biosystems, Foster City, CA, USA). The monkey $18 \mathrm{~s}$ gene was used as an internal marker. The gene expression level was calculated by its individual standard curve, and then normalized to $18 \mathrm{~s}$ level for each sample. The primers and probe sequences $\left(5^{\prime}-3^{\prime}\right)$, designed against macaque mRNA for all the target genes, were purchased from Applied Biosystems and Invitrogen (Carlsbad, CA, USA), including endothelial nitric oxide synthase (eNOS) (Hs00167166_ml), plasminogen activator inhibitor-1 (PAl-1) (Hs01126607_g1), tissue-type plasminogen activator (t-PA) (For: TGACTGCTACCTTGGGAATGG; Rev: TGCCTATCAGCATCAT GGAATT; Probe: CACAGCCTCACCGCATCGGGT), VCAM-1 (vascular cell adhesion molecule-1) (For: TGAGAGGCAGACTTCCCTGAAT; Rev: TTाTCAGGCAGCAAGT TाTCC; Probe: CACCTATGCCCCTTGCTCTGAGCAAG), intercellular adhesion molecule-1 (ICAM-1) (For: TAGCCGGCCAGCTTGTACAC; Rev: TGGCCACGTCCA GTTCC; Probe: TCCGTGTCCTGTATGGACCCCGA), vascular endothelial growth factor (VEGF) (For: GAAGTGGTGAAGTTCATGGATGTC; Rev: CACCAGGGTCTC GATTGGAT; Probe: ATCAGCGCAGCTACT), tumor necrosis factor $\alpha$ (TNF $\alpha$ ) (For: CTCTGGCTCAGGCAGTCAGAT; Rev: CATGGGCTACAGGCTTGTCA, Probe: ATCTTCTCGAACCCC), MCP-1 (monocyte chemotactic protein-1) (For: AGGCTGGCGAGCTATAGAAGAAT; Rev: TCTTGAAGATCACAGCTTCTIT GG; Probe: ACCAGCAGCAAGTGT) and IL-6 (interleukin 6) (For: CAGATATGA ACTCCGTCTCCACAA; Rev: AGCAGCCCCAGGGAGAAG; Probe: CGCCTTCGGT CCAGTT). 
Hematoxylin/eosin staining for morphological assessment H\&E (hematoxylin/eosin)-stained paraffin-sliced sections of abdominal aorta tissue from $\mathrm{C} / \mathrm{C}$ and $\mathrm{H} / \mathrm{H}$ animals were used for the morphological assessment, specifically the changes underneath the endothelium monolayer. Pictures were then taken using Slidebook5.0 (Intelligent Imaging Innovations, Inc., Denver, CO, USA).

Immunohistochemistry for measurement of intima thickness Paraffin-sliced sections containing abdominal aorta rings were rehydrated, followed by antigen retrieval. Then, sheep polyclonal anti- $\mathrm{CD}_{31}$ primary antibody (1:400, \#AF806, R\&D Systems Inc., Minneapolis, MN, USA) was applied after blocking and left overnight. The next day, sections were incubated with the secondary antibody, followed by $A / B$ solution and Nickel DAB solution treatment. Finally, the sections were covered with a strip of Permount and left to dry for photographing. Intima thickness of the abdominal aorta rings was then measured as the distance between the endothelium monolayer and the elastic tissue underneath, using the Leica Application Suite (version 3.5.0 software, Buffalo Grove, IL, USA). Three rings from each aorta were analyzed with eight equally spaced measurements. Therefore, 24 total measurements yielded an average value for each animal, which was used for the final calculations and statistical comparisons.

\section{Statistical analysis}

Data were expressed as mean \pm s.e. Statistical comparisons of the cumulative percentage of relaxation under different treatments were performed by two-way analysis of variance, followed by Bonferroni's test. $\mathrm{EC}_{50}$ value, gene expression level and intima thickness comparisons for various groups of animals were performed by one-way analysis of variance, followed by Bonferroni's multiple comparison test analysis. Statistical comparisons between two groups were performed by $t$-tests. Correlative relationships were analyzed by Pearson's correlation analysis. A $P$-value $<0.05$ was considered statistically significant.

\section{RESULTS}

Metabolic phenotype

At the time of necropsy (13 months of age), there was no difference in body weight between groups (Table 1). The total body fat was $<3 \%$ for all animals. Despite the lean phenotype, specifically in $\mathrm{H} / \mathrm{H}$ offspring, there was an elevation in plasma fasting insulin level, as well as glucose-stimulated insulin secretion (IAUC) and HOMA-IR in comparison with $\mathrm{C} / \mathrm{C}$, indicating that $\mathrm{H} / \mathrm{H}$ juveniles were developing insulin resistance. However, fasting glucose values were normal in these offspring. There was also no difference between groups in fasting free fatty acids and triglycerides. Importantly, the animals exposed to the HFD only during the postweaning period $(\mathrm{C} / \mathrm{H})$ and the postweaning diet intervention group $(\mathrm{H} / \mathrm{C})$ displayed intermediate values for fasting insulin, IAUC and HOMA-IR, that were not significantly different from either the $\mathrm{C} / \mathrm{C}$ or $\mathrm{H} / \mathrm{H}$ groups.

\section{ACh-induced endothelium-dependent vasorelaxation response}

The measurement of ACh-induced endothelium-dependent vasorelaxation response is the most useful test of endothelial function. Thus, in this study, we investigated the effect of maternal and early postnatal HFD exposure on endothelium-dependent vasorelaxation of the offspring by performing ACh-induced concentration-relaxation response on both abdominal aorta and renal artery rings.

As shown in Figure $1 \mathrm{a}$, the maximal vasorelaxation response (Rmax) produced by ACh was remarkably decreased in abdominal aorta rings taken from $\mathrm{H} / \mathrm{H}$ juveniles $(44.6 \pm 6.2 \%, n=10)$ compared with C/C $(79.0 \pm 7.8 \%, n=9)$, along with a $>300 \%$ higher $E_{50}$ value for the corresponding relaxation curve $(-7.102 \pm 0.143$ vs $-7.634 \pm 0.103$ LogM, $P<0.01$; Figure $1 \mathrm{~b})$. On the contrary, $\mathrm{C} / \mathrm{H}$ juveniles that have a similar adiposity, demonstrated an unchanged vascular relaxation response, whereas the $\mathrm{H} / \mathrm{C}$ animals completely reversed the depressed vasodilatation. Concurrently, the $\mathrm{EC}_{50}$ value against the curve for $\mathrm{H} / \mathrm{C}$ animals was $11-$ fold lower in comparison with $\mathrm{H} / \mathrm{H}$ juveniles $(-8.170 \pm 0.280$ LogM vs $-7.102 \pm 0.143)$. Furthermore, the vessels of $\mathrm{H} / \mathrm{C}$ animals tended to be more sensitive in response to $\mathrm{ACh}$, with a slightly left-shifted curve in comparison with $\mathrm{C} / \mathrm{C}$. Nevertheless, $\mathrm{EC}_{50}$ values against the $\mathrm{ACh}$-induced vasorelaxant curve for juveniles exposed to the maternal HFD $(\mathrm{H} / \mathrm{H}$ and $\mathrm{H} / \mathrm{C})$ were demonstrated to be positively correlated with IAUC $(r=0.53$ $P<0.05$; Figure 1c). These data suggest that maternal HFD exposure contributes to the detrimental effect of insulin resistance on vasorelaxant function in offspring.

Noteworthy, all of the tested abdominal aorta rings were under similar pre-contraction forces induced by thromboxane $A_{2}$ analog $\mathrm{U}_{46619}$ before the vasorelaxation responses were elicited (Figure 1d).

Interestingly, there was no difference in renal artery endothelial responses $(83.4 \pm 8.6 \%, 82.1 \pm 7.4 \%, 77.0 \pm 11.2 \%$ and $57.3 \pm 6.4 \%, n=4-9$; Supplementary Figure S1) between any of the groups.

\section{eNOS gene expression}

eNOS is of critical importance in ACh-induced endotheliumdependent vasodilatation. In order to determine whether the depressed ACh-induced vasorelaxation response is due to the depressed expression of eNOS in the vessel, we measured the eNOS mRNA level in abdominal aorta tissue from various groups of juvenile offspring, using quantitative real-time polymerase

Table 1. Metabolic phenotype for the Juvenile offspring

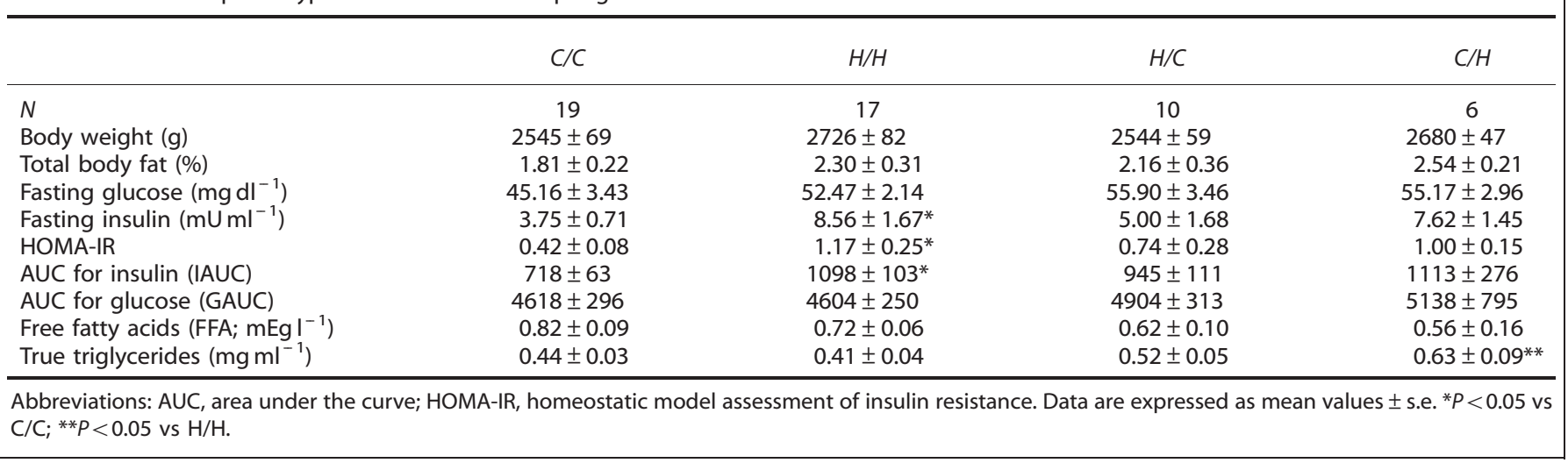


a
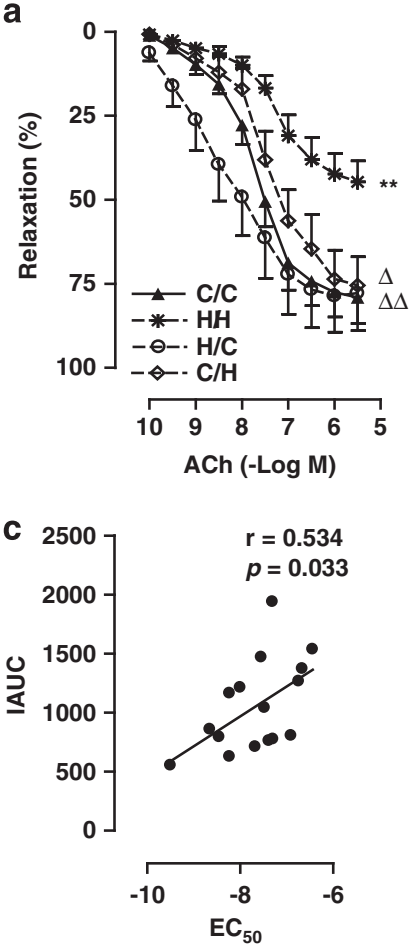

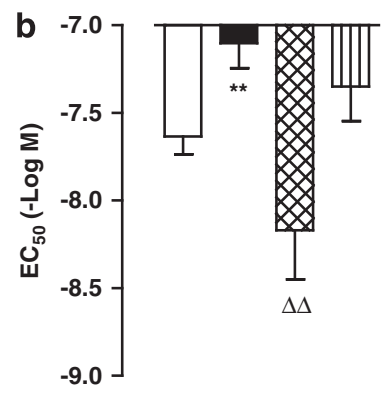

$\mathrm{C} / \mathrm{C} \mathrm{H/H} \mathrm{H/C} \mathrm{C/H}$

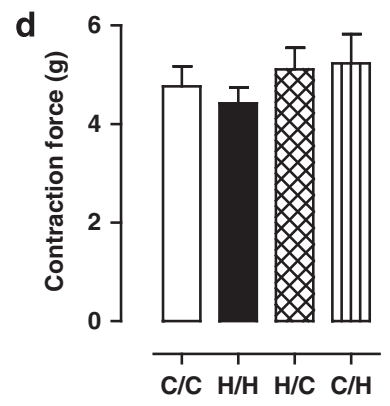

Figure 1. ACh-induced endothelium-dependent vasorelaxation response in abdominal aorta tissue. (a) Cumulative concentrationrelaxation (\%) curves for $\mathrm{ACh}(-10$ to -5.5 LogM) in abdominal aorta rings against $U_{46619}$ (9,11-dideoxy-11 $\alpha, 9 \alpha$-epoxy-methanoprostaglandin $\mathrm{F}_{2 \alpha}$ ). (b) Corresponding $\mathrm{EC}_{50}$ value for each curve. (c) Correlative relationship between $\mathrm{EC}_{50}$ and IAUC for juveniles born to HFD consumption dams. (d) Contracting forces against $U_{46619}$ Values were expressed as mean \pm s.e., $n=6-10$. ${ }^{* *} P<0.01$ vs $C / C$, ${ }^{\Delta} P<0.05$ and ${ }^{\Delta}{ }_{P}<0.01$ vs $\mathrm{H} / \mathrm{H}$.

chain reaction. As shown in Supplementary Figure S2a, the level of eNOS mRNA in the intact abdominal aorta was not different between groups. However, it was positively correlated with Rmax for $\mathrm{H} / \mathrm{H}$ group of animals ( $r=0.72, P<0.05$; Supplementary Figure $\mathrm{S} 2 \mathrm{~b}$ ).

\section{Intima thickness}

During the development of atherosclerosis, more connective tissues build up in intima of the vessel. In the current study, the mean intima thickness of the abdominal aorta for $\mathrm{H} / \mathrm{H}$ animals was nearly $90 \%$ higher than that for $C / C(P<0.01$; Figure 2a), accompanied by the predominant proliferation of smooth muscle cells deposited underneath the endothelium monolayer (Figure 2b). Abnormal increase in the intima wall was also observed in diet-switched $\mathrm{H} / \mathrm{C}$ and $\mathrm{C} / \mathrm{H}$ animals $(P<0.05$; Figure 2a).

\section{Pro-inflammation}

On H\&E slides, vacuole-like cells were readily apparent, depositing underneath the endothelium in abdominal aorta rings specifically in $\mathrm{H} / \mathrm{H}$ juveniles. However, inflammation with obvious histiocytic infiltration could not yet be detected at this stage (Figure 3 ). Nonetheless, proinflammatory markers such as VEGF, TNF $\alpha$ and ICAM-1 in aortas from $\mathrm{H} / \mathrm{H}$ animals were increased in comparison to $C / C$ offspring $(P<0.05, P<0.01)$. Postweaning diet reversal $(H / C)$ did not significantly reverse the expression of VEGF or TNF $\alpha$ (Figure 4a). However, ICAM- 1 tends to be lower in the H/C animals compared with $\mathrm{H} / \mathrm{H}$, but it was not significantly different from either the $\mathrm{C} / \mathrm{C}$ or $\mathrm{H} / \mathrm{H}$ group. The $\mathrm{C} / \mathrm{H}$ animals displayed a modest,

but significant increase in the expression of VEGF, but had normal expression levels for TNF $\alpha$ and ICAM-1 (Figure 4a).

There was no significant difference in the expression of IL-6, VCAM-1 and MCP-1 between any of these groups (Figure 4b). Furthermore, none of these cytokines was differently expressed in the renal artery from any group (Supplementary Figure S3).

\section{Fibrinolytic system}

Endothelial dysfunction usually occurs in conjunction with impaired fibrinolysic function, predisposing the vessel to a precoagulant state. $^{36,37}$ In the current study, we observed an increased expression of PAI-1 in abdominal aorta tissue from $\mathrm{H} / \mathrm{H}$ juveniles in comparison with $C / C(P<0.01)$, along with a notable decrease in the ratio of t-PA relative to PAl-1 $(P<0.01)$, indicating a disrupting balance between fibrinolysis and coagulation of the vessel (Figure 5). No significant difference in PAI-1 expression was detected for both $\mathrm{H} / \mathrm{C}$ and $\mathrm{C} / \mathrm{H}$ groups compared with either $\mathrm{C} / \mathrm{C}$ or $\mathrm{H} / \mathrm{H}$; however, the t-PA/PAl- 1 ratio was significantly higher in the $\mathrm{H} / \mathrm{C}$ and $\mathrm{C} / \mathrm{H}$ groups compared with the $\mathrm{H} / \mathrm{H}$, but was not different from the $\mathrm{C} / \mathrm{C}$ group. No notable differences were detected in renal arteries (Supplementary Figure S4).

Of note, there were no sex differences regarding the manifestation of vascular function.

\section{DISCUSSION}

Among all the animal models, NHP have the greatest similarity to humans with obesity and/or diabetes in terms of the alterations in plasma glucose level and pathologic features. It is also the closest animal model for investigating the complications of metabolic diseases, such as cardiovascular disorders. In our NHP model, vascular function of the offspring was impaired due to maternal HFD exposure, manifested as depressed endothelium-dependent vasodilatation, thickened intima wall, as well as the development of inflammation and prothrombosis. Such manifestation increases the susceptibility of the offspring to an increased risk of earlyonset atherogenesis. Importantly, the vascular function impairments observed in HFD offspring were before the development of obesity. Based on our findings, it is clear that both earlyprogramming events and postweaning diet contribute to the homeostasis of endothelial function. It is equally clear that the detrimental effect on endothelial function due to maternal HFD consumption can be partially reversed by a healthy diet intervention.

Maternal and early postnatal HFD exposure gives rise to an offspring phenotype of endothelial dysfunction

It is recognized that endothelium-dependent vasodilatation is a key measurement of endothelial function. Commonly, impaired arteries have a decreased capacity to dilate fully in response to certain physiological/pharmacological stimuli. Our findings on the NHP model showed that the abdominal aorta rings taken from $\mathrm{H} / \mathrm{H}$ juveniles lost nearly $50 \%$ of dilation capacity, with a potency that was more than threefold lower in response to the endothelium-dependent vasodilator ACh. The blunted vasodilatation response in the $\mathrm{H} / \mathrm{H}$ NHP offspring is consistent with previous studies from obese adult rat offspring born to dams fed a fat-rich diet. $^{13,14}$ However, these earlier studies focused on adult offspring rats that had increased adiposity. Our studies demonstrate that the endothelial dysfunction occurs in young lean animals $(<3 \%$ body fat) with similar body weights. Furthermore, our results indicate that while the metabolic phenotype is more severe in males than in females (unpublished observations), there was no sexual dimorphism in the abnormalities of the vascular function. Interestingly, the vasodilation of the renal artery rings taken from the same $\mathrm{H} / \mathrm{H}$ juveniles did not present similar diminished vasodilatation as those in abdominal aorta rings. Although the 


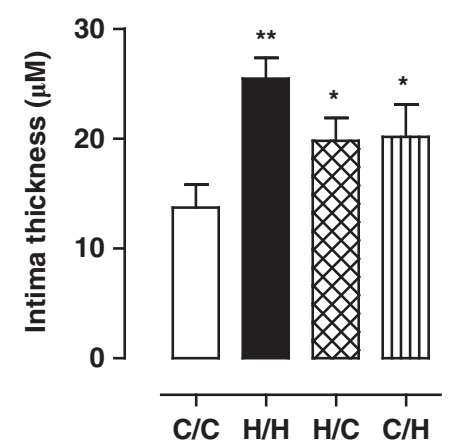

b
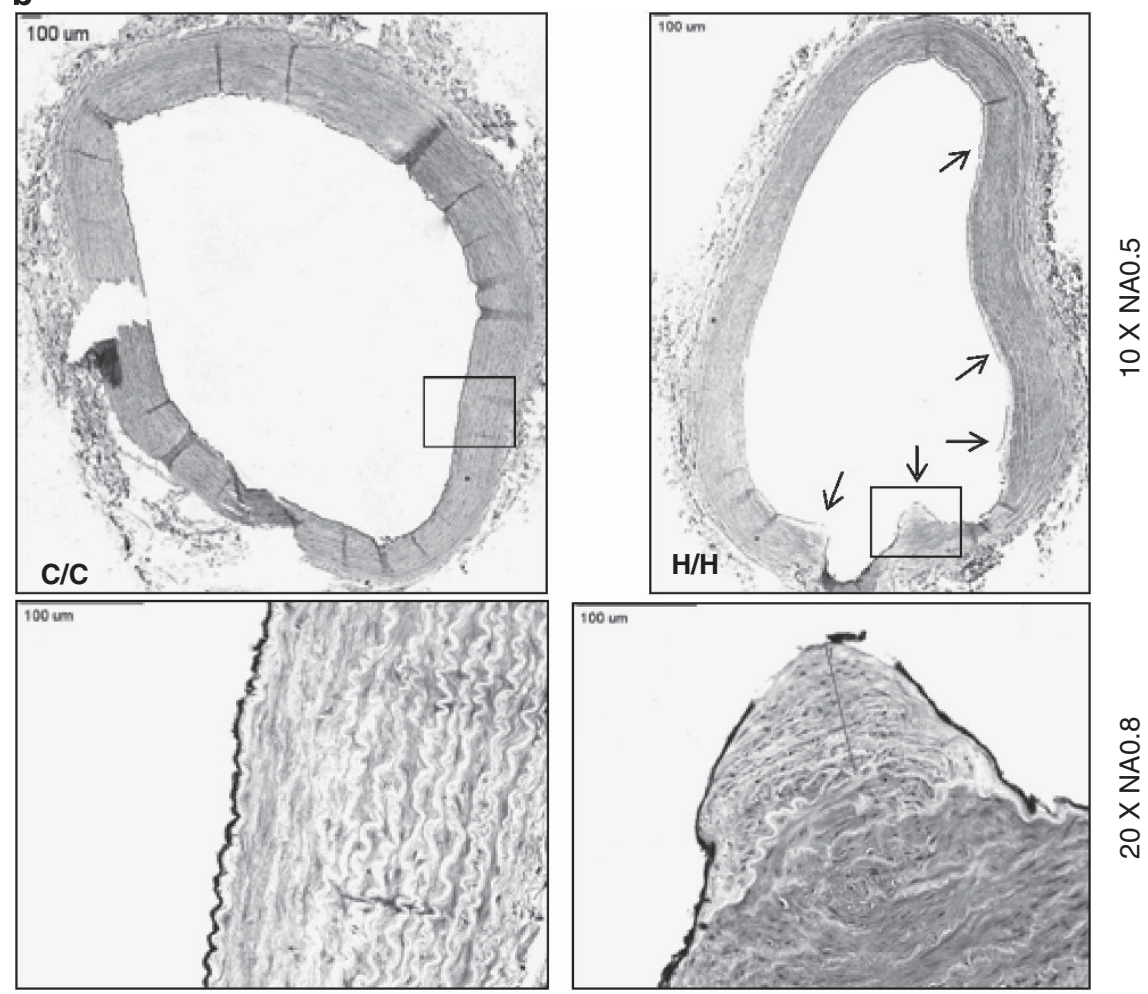

Figure 2. Intima thickness of the abdominal aorta tissue. (a) Mean intima thickness value of abdominal aorta tissue for various groups of juvenile offspring. (b) Immunohistochemistry pictures immunostained with endothelial marker $\mathrm{CD}_{31}$, typical showing the intima thickness for abdominal aorta taken from $\mathrm{C} / \mathrm{C}$ and $\mathrm{H} / \mathrm{H}$ animals. Arrows indicate areas of intima thickening, and the top boxes indicate areas of enlarged pictures. Values were expressed as mean \pm s.e., $n=3-5$. ${ }^{*} P<0.05$ and ${ }^{*} P<0.01$ vs $C / C$.

present study could not clarify whether the discrepancy of the vasorelaxation response of these two different arteries was related to the difference in the vascular structure and function, the results of this study reflect a different pathophysiological alteration in endothelial function related to insulin resistance. While it has been shown that insulin resistance is related more closely to macrovasculature than micro-vasculature, ${ }^{38}$ our findings demonstrate a vessel-specific dysfunction difference in macro vessels.

There are several possibilities that could contribute to endothelial dysfunction in the aorta, including decreased expression of eNOS, an increased diffusion barrier with impaired endothelial signal transduction caused by increased intima thickness, lower bioactivity of eNOS-derived nitric oxide (NO) by reactive oxygen species, reduced availability of L-arginine, or reduced response of vascular smooth muscle to NO. ${ }^{39}$ Among these, the last two possibilities are less likely, as the endothelial cells contain concentrations of L-arginine hundreds of times greater than those required for the activity of eNOS to synthesize NO (micromolar range). ${ }^{40,41}$ Abnormalities in vasomotion of the vascular smooth muscle are less likely to develop until the advanced stage of atherosclerosis. ${ }^{42}$ Thus, the likely major contributors are depressed eNOS expression, thickened intima or decreased bioactivity of NO. In our current studies, we observed no differences in eNOS expression in the $\mathrm{H} / \mathrm{H}$ aorta, suggesting that the overall endothelial dysfunction in the aorta of these animals was not significantly affected by the expression level of eNOS. Nonetheless, within the $\mathrm{H} / \mathrm{H}$ group, the maximal relaxant capacity of the abdominal aorta rings was positively correlated with the eNOS expression level, indicating that eNOS may have a minor contribution to endothelial dysfunction in our model.

Thickened intima, commonly presented during the process of atherogenesis, increases the diffusion barrier of the endotheliumderived factors from endothelial cells to the smooth muscle cells underneath and consequently impairs signal transduction in the vasculature. Therefore, the outcome of the greatly increased intima thickness observed in the abdominal aorta vascular ring can be a contributor to the notable depression in vasodilatation capacity found in juvenile $\mathrm{H} / \mathrm{H}$ offspring. The decreased bioactivity 


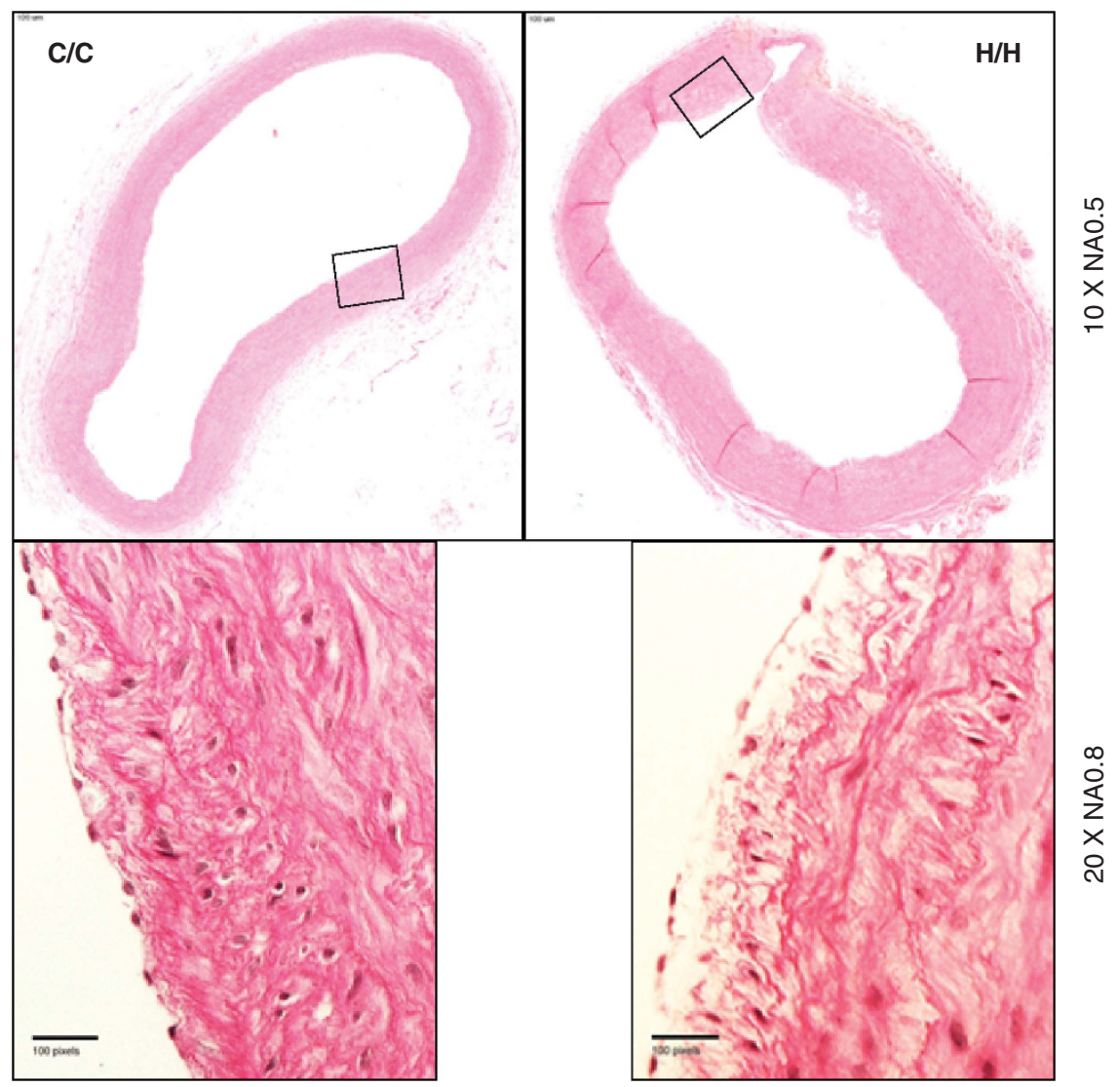

Figure 3. Morphological assessment of the abdominal aorta tissue. H\&E staining pictures typically showing the different morphological manifestation for the abdominal aorta rings taken from animals from $\mathrm{C} / \mathrm{C}$ and $\mathrm{H} / \mathrm{H}$. Top boxes indicate areas of enlarged pictures below.

of NO, which contributes to endothelial impairment, could be caused by increased oxidative stress due to excess generation of reactive oxygen and nitrogen species, such as superoxide anion and peroxynitrite. The increase in reactive oxygen species might come from elevated glucose that causes more superoxide generation, combined with the low oxygen diffusion associated with increased wall thickness. The lower bioactivity of NO weakens its anti-proliferative and anti-inflammatory properties. ${ }^{43,44}$ In the present study, we observed an obvious proliferation of smooth muscle cells deposited underneath the endothelium, which hints at impairment in the bioactivity of NO. In addition, even though definite inflammation with histiocytic infiltration under the endothelium was not observed, elevated expression levels of proinflammatory factors, such as VEGF, TNF $\alpha$ and ICAM-1 were detected in aorta tissue from $\mathrm{H} / \mathrm{H}$ juveniles. Highly expressed proinflammatory cytokines and chemokines usually occur at the early stage of inflammation in the vessel. ${ }^{45,46}$ Vascular inflammation has a pivotal role in all stages of atherosclerosis, from initiation through progression, and, ultimately, to the occurrence of thrombotic complications. ${ }^{47,48}$ Atherosclerosis is also thus regarded as a dynamic and progressive pathophysiological process arising from a combination of inflammation and endothelial dysfunction, both of which were observed in the aorta of our $\mathrm{H} / \mathrm{H}$ offspring.

Decreased bioactivity of NO not only undermines its properties of anti-inflammation and anti-proliferation, it also weakens its ability to prevent the development of coagulation. ${ }^{43} \mathrm{~A}$ vascular pro-thrombotic state is commonly manifested as destroyed fibrinolytic function. Fibrinolysis is the process wherein the blood clots produced by coagulation are broken down. It is usually initiated by the activation of plasmin, which could be activated in response to t-PA, whereas t-PA itself could be inhibited by PAl. As observed in our research, excess levels of PAI-1 released from the impaired endothelium could inhibit the activation of plasmin and thereby decrease the break-down of the fibrin mass. This ultimately destroys the balance between fibrinolysis and coagulation in the vessel, thereby promoting atherogenesis. For $\mathrm{H} / \mathrm{H}$ juveniles, the up-regulated expression of PAl-1, together with notably depressed ratio of t-PA/PAI-1, indicated impaired fibrinolytic function of the abdominal aorta. These findings are consistent with other studies that show the coexistence of hypercoagulability with insulin resistance. ${ }^{36,37}$

Although insulin resistance links obesity to generalized metabolic disorders, and endothelial dysfunction is the main feature of both metabolic disorders and CVD, it is disputed whether the occurrence of insulin resistance promotes the induction of endothelial dysfunction or whether abnormal endothelial function exacerbates insulin resistance. ${ }^{49-51}$ In any case, insulin resistance is unlikely to promote atherogenesis independently of other risk factors. More evidence is required to uncover the role of insulin resistance in endothelial dysfunction. However, our data clearly show that endothelial dysfunction and insulin resistance were coexistent, as demonstrated by impaired endothelium function concurrent with elevated fasting insulin levels, IAUC and HOMA-IR. Moreover, a positive correlation between $\mathrm{EC}_{50}$ of $\mathrm{ACh}$-induced vasodilatation and IAUC was observed in the HFD offspring.

Postweaning HFD exposure moderately elevates the susceptibility to abnormalities in vascular endothelial function

In order to understand whether the high-fat dietary intake itself, or whether early programming events contribute to the abnormal 
a

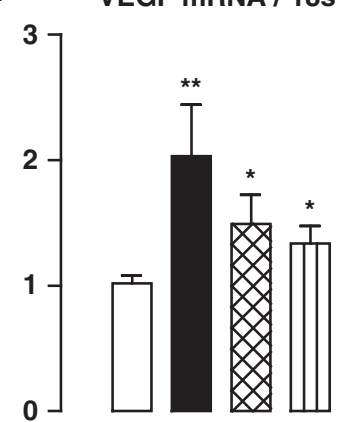

$\mathrm{C} / \mathrm{C} \mathrm{H} / \mathrm{H} \mathrm{H} / \mathrm{C} \mathrm{C} / \mathrm{H}$

b IL-6 mRNA / 18s

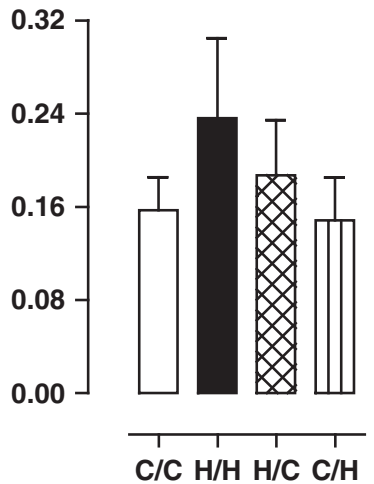

TNF $\alpha$ mRNA / 18s

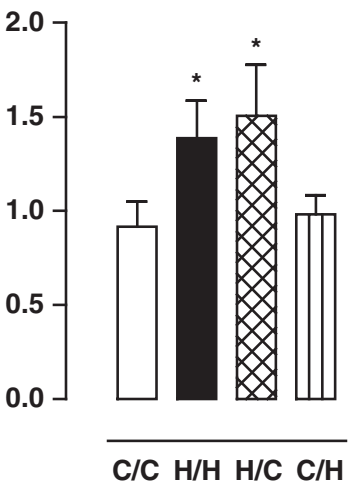

VCAM-1 mRNA / 18s

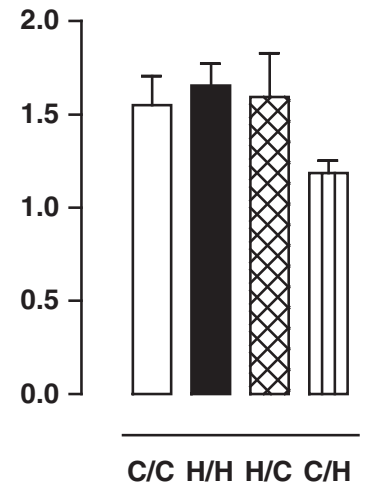

ICAM-1 mRNA / 18s

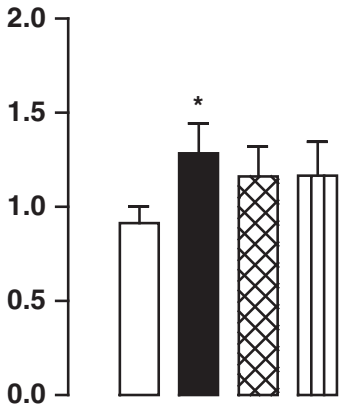

$\mathrm{C} / \mathrm{CH} / \mathrm{H} \mathrm{H} / \mathrm{C} \mathrm{C/H}$

MCP-1 mRNA / 18S

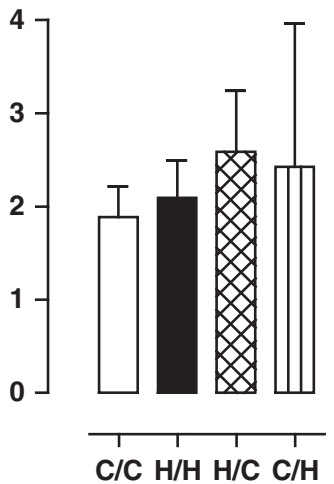

Figure 4. Expression of proinflammatory factors in abdominal aorta tissue. (a) Relative expression levels of VEGF, TNF $\alpha$ and ICAM-1, in abdominal aorta tissue of Juvenile offspring. (b) Relative expression levels of IL-6, VCAM-1 and MCP-1 in abdominal aorta tissue of Juvenile offspring. Values were expressed as mean \pm s.e., $n=6-17$. ${ }^{*} P<0.05$ and ${ }^{*} P<0.01$ vs $C / C$.

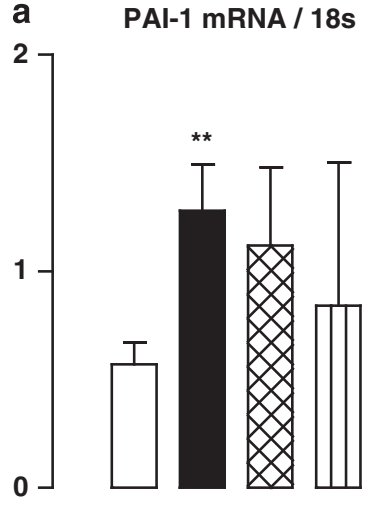

$\mathrm{C} / \mathrm{C} \quad \mathrm{H} / \mathrm{H} \quad \mathrm{H} / \mathrm{C} \quad \mathrm{C} / \mathrm{H}$ b

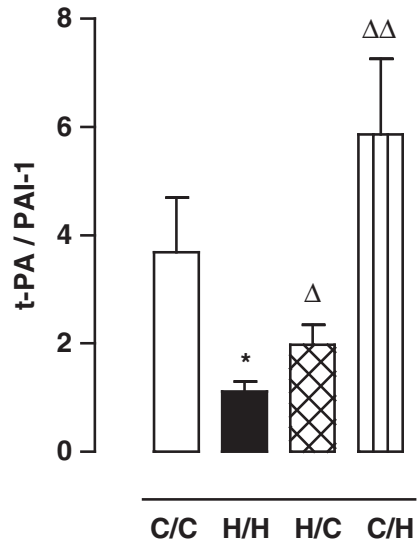

Figure 5. Expression of fibrinolytic factors in abdominal aorta tissue. (a) Relative expression level of PAI-1 and (b) the ratio between t-PA and PAI-1 in abdominal aorta tissue of juvenile offspring. Values were expressed as mean \pm s.e., $n=6-17 .{ }^{*} P<0.05$, ${ }^{* *} P<0.01$ vs $C / C$ and ${ }^{\Delta} P<0.05,{ }^{\Delta \Delta} P<0.01$ vs $\mathrm{H} / \mathrm{H}$.

endothelial function in $\mathrm{H} / \mathrm{H}$ animals, we observed the endothelial function in offspring with HFD ingestion limited to the postweaning period $(\mathrm{C} / \mathrm{H})$. It is interesting that despite a similar metabolic phenotype in $\mathrm{H} / \mathrm{H}$ and $\mathrm{C} / \mathrm{H}$ animals, abnormal vasodilatation in the aorta was only observed in $\mathrm{H} / \mathrm{H}$ offspring. However, there is evidence of emerging vascular dysfunction as the intima of the vessel of $\mathrm{C} / \mathrm{H}$ offspring was thickened, and the cytokine VEGF

expression was elevated as compared with $\mathrm{C} / \mathrm{C}$. In view of these findings, the endothelial dysfunction demonstrated in the young $\mathrm{H} / \mathrm{H}$ offspring is primarily in response to early programming events, rather than simply an effect of current diet. However, it is highly likely that continued HFD exposure in the $\mathrm{C} / \mathrm{H}$ group would likely eventually result in more extensive endothelial dysfunction.

Diet intervention after weaning partially improves endothelial dysfunction

If maternal HFD consumption during pregnancy and early postnatal period resulted in endothelial dysfunction in the offspring, then it is important to know whether the damage is reversible. In order to address this critical question, some of our HFD offspring underwent a diet switch to a normal chow after weaning $(\mathrm{H} / \mathrm{C})$. Importantly, there was no obvious difference in ACh-induced vasorelaxation curve detected in aorta rings in $\mathrm{H} / \mathrm{C}$ juveniles compared with $\mathrm{C} / \mathrm{C}$, suggesting at least some improvement was produced in the diet intervention group. In fact, the vessels of these $\mathrm{H} / \mathrm{C}$ animals displayed higher sensitivity in response to $\mathrm{ACh}$. This was surprising and the underlying causes of this hypersensitivity are unknown. However, there was still evidence that increased intima wall and elevated pro-inflammation cytokine expression persisted in the $\mathrm{H} / \mathrm{C}$ animals, suggesting that the diet intervention was not sufficient to completely normalize the damage caused by early HFD exposure. A number of causes could explain these findings: they could be the result of the relatively short time period of diet intervention, or as a consequence of the programming events that have already altered certain genotypes, or both. The relatively normal manifestation of the metabolic parameters, such as body weight, 
fasting insulin level and HOMA-IR value in $\mathrm{H} / \mathrm{C}$ juveniles, indicates that metabolic disorders tend to be easily rehabilitated even within a short period of time.

Taken together, our data demonstrate that endothelial dysfunction may be linked to both early programming events and environmental contributions such as dietary consumption, independent of a significant metabolic phenotype. Also, our findings indicate that postweaning dietary intervention improves the offspring's vascular function through reducing exposure to the detrimental effects associated with HFD consumption. Based on these findings, we are currently investigating whether the adult NHP offspring with maternal HFD exposure will be at a higher risk to develop substantial diabetes, hypertension and other metabolic-associated disorders; and whether the adult offspring with the longer-term diet modification will experience a complete normalization of the metabolic and cardiovascular disorders.

\section{CONFLICT OF INTEREST}

The authors declare no conflict of interest.

\section{ACKNOWLEDGEMENTS}

We gratefully acknowledge our NIH funding sources R01-DK079194 (to KL Grove), P51OD011092 (to KL Grove and ONPRC) and P01-HD034430 (to KL Thornburg and KL Grove). Also, we express our profound gratitude to the Division of Animal Resources and Veterinary staff at ONPRC for their technical assistance with the animals.

\section{REFERENCES}

1 Han JC, Lawlor DA, Kimm SY. Childhood obesity. Lancet 2010; 375: 1737-1748.

2 Barker DJ, Gluckman PD, Godfrey KM, Harding JE, Owens JA, Robinson JS. Fetal nutrition and cardiovascular disease in adult life. Lancet 1993; 341: $938-941$.

3 Barker DJ. Fetal origins of cardiovascular disease. Ann Med 1999; 31 (Suppl 1): 3-6.

4 Barker DJ. Fetal programming of coronary heart disease. Trends Endocrinol Metab 2002; 13: $364-368$.

5 Strauss RS. Effects of the intrauterine environment on childhood growth. Br Med Bull 1997; 53: 81 - 95 .

6 Ravelli AC, Der Meulen JH, Osmond C, Barker DJ, Bleker OP. Obesity at the age of $50 \mathrm{y}$ in men and women exposed to famine prenatally. Am J Clin Nutr 1999; 70: 811-816.

7 McMillen IC, Robunson JS. Developmental origins of the metabolic syndrome: prediction, plasticity, and programming. Physiol Rev 2005; 85: 571-633.

8 Le Clair C, Abbi T, Sandhu H, Tappia PS. Impact of maternal undernutrition on diabetes and cardiovascular disease risk in adult offspring. Can J Physiol Pharmacol 2009; 87: 161 - 179 .

9 Vonnahme KA, Hess BW, Hansen TR, McCormick RJ, Rule DC, Moss GE et al. Maternal undernutrition from early- to mid-gestation leads to growth retardation, cardiac ventricular hypertrophy, and increased liver weight in the fetal sheep. Biol Reprod 2003; 69: 133-140.

10 Gopalakrishnan GS, Gardner DS, Dandrea J, Langley-Evans SC, Pearce S, Kurlak LO et al. Influence of maternal pre-pregnancy body composition and diet during early-mid pregnancy on cardiovascular function and nephron number in juvenile sheep. Br J Nutr 2005; 94: 938-947.

11 Morrison JL, Botting KJ, Dyer JL, Williams SJ, Thornburg KL, McMillen IC. Restriction of placental function alters heart development in the sheep fetus. Am J Physiol Regul Integr Comp Physiol 2007; 293: R306-R313.

12 Louey S, Jonker SS, Giraud GD, Thornburg KL. Placental insufficiency decreases cell cycle activity and terminal maturation in fetal sheep cardiomyocytes. J Physiol 2007; 580 (Part 2): 639-648.

13 Armitage JA, Lakasing L, Taylor PD, Balachandran AA, Jensen RI, Dekon V et al. Developmental programming of aortic and renal structure in offspring of rats fed fat-rich diets in pregnancy. J Physiol 2005; 565: 171-184.

14 Taylor PD, McConnell J, Khan IY, Holemans K, Lawrence KM, Asare-Anane $\mathrm{H}$ et al. Impaired glucose homeostasis and mitochondrial abnormalities in offspring of rats fed a fat-rich diet in pregnancy. Am J Physiol Regul Integr Comp Physiol 2005; 288: R134-R139.

15 Samuelsson AM, Matthews PA, Argenton M, Christie MR, McConnell JM, Jansen EH et al. Diet-induced obesity in female mice leads to offspring hyperphagia, adiposity, hypertension, and insulin resistance: a novel murine model of developmental programming. Hypertension 2008; 51: 383-392.
16 McCurdy CE, Bishop JM, Williams SM, Grayson BE, Smith SM, Friedman JE et al. Maternal high fat diet triggers lipotoxicity in the fetal liver of the nonhuman primate. J Clin Invest 2009; 19: 323-335.

17 Frias $A E$, Morgan TK, Evans AE, Rasanen J, Oh KY, Thornburg KL et al. Maternal high-fat diet disturbs uteroplacental hemodynamics and increases the frequency of stillbirth in a Nonhuman Primate model of excess nutrition. Endocrinology 2011; 152: $2456-2464$.

18 Sullivan EL, Grayson B, Takahashi D, Robertson N, Maier A, Bethea CL et al. Chronic consumption of a high-fat diet during pregnancy causes perturbations in the serotonergic system and increased anxiety-like behavior in nonhuman primate offspring. J Neurosci 2010; 30: 3826-3830.

19 Grayson BE, Levasseur PR, Williams SM, Smith MS, Marks DL, Grove KL. Changes in melanocortin expression and inflammatory pathways in fetal offspring of nonhuman primates fed a high-fat diet. Endocrinology 2010; 151: 1622-1632.

20 Suter M, Bocock P, Showalter L, Hu M, Shope C, McKnight R et al. Epigenomics: maternal high-fat diet exposure in utero disrupts peripheral circadian gene expression in nonhuman primates. FASEB J 2011; 25: 714-726.

21 Cox J, Williams S, Grove KL, Lane RH, Aagaard-Tillery KM. A maternal high-fat diet is accompanied by alterations in the fetal primate metabolome. Am J Obstet Gynecol 2009; 201: 281.e1-9.

22 Meyers MR, Gokce N. Endothelial dysfunction in obesity: etiological role in atherosclerosis. Curr Opin Endocrinol Diabetes Obes 2007; 14: 365-369.

23 Poirier P, Eckel RH. Obesity and cardiovascular disease. Curr Atheroscler Rep 2002; 4: $448-453$.

24 Poirier P, Giles TD, Bray GA, Hong Y, Stern JS, Pi-Sunyer FX et al. Obesity and cardiovascular disease: pathophysiology, evaluation, and effect of weight loss: an update of the 1997 American Heart Association Scientific Statement on Obesity and Heart Disease from the Obesity Committee of the Council on Nutrition, Physical Activity, and Metabolism. Circulation 2006; 113: 898-918.

25 Davignon J, Ganz P. Role of endothelial dysfunction in atherosclerosis. Circulation 2004; 109: III27-III32.

26 Lerman A, Zeiher AM. Endothelial function: cardiac events. Circulation 2005; 111: $363-368$.

27 Stepp DW. Impact of obesity and insulin resistance on vasomotor tone: nitric oxide and beyond. Clin Exp Pharmacol Physiol 2006; 33: 407-414.

28 Tabit CE, Chung WB, Hamburg NM, Vita JA. Endothelial dysfunction in diabetes mellitus: molecular mechanisms and clinical implications. Rev Endocr Metab Disord 2010; 11: $61-74$.

29 Bakker W, Eringa EC, Sipkema P, van Hinsbergh VW. Endothelial dysfunction and diabetes: roles of hyperglycemia, impaired insulin signaling and obesity. Cell Tissue Res 2009; 335: 165-189.

30 Calles-Escandon J, Cipolla M. Diabetes and endothelial dysfunction: a clinical perspective. Endocr Rev 2001; 22: 36-52.

31 Meyers MR, Gokce N. Endothelial dysfunction in obesity: etiological role in atherosclerosis. Curr Opin Endocrinol Diabetes Obes 2007; 14: 365-369.

32 Damjanovic M, Barton M. Food intake and cardiovascular response. Curr Hypertens Rep 2008; 10: $25-31$.

33 Solnica B, Naskalski JW. Quality of SMBG in clinical practice. Scand J Clin Lab Invest Suppl 2005; 240: $80-85$.

34 Fan L, Yang Q, Xiao XQ, Grove KL, Huang Y, Chen ZW et al. Dual actions of cilnidipine in human internal thoracic artery: inhibition of calcium channels and enhancement of endothelial nitric oxide synthase. J Thorac Cardiovasc Surg 2011; 141: $1063-1069$.

35 He GW, Fan L, Furnary A, Yang Q. A new antispastic solution for arterial grafting: nicardipine and nitroglycerin cocktail in preparation of internal thoracic and radial arteries for coronary surgery. J Thorac Cardiovasc Surg 2008; 136: 673-680, 680.e1-2.

36 Kakafika Al, Liberopoulos EN, Karagiannis A, Athyros VG, Mikhailidis DP. Dyslipidaemia, hypercoagulability and the metabolic syndrome. Curr Vasc Pharmacol 2006; 4: $175-183$.

37 Van Gaal LF, Mertens IL, De Block CE. Mechanisms linking obesity with cardiovascular disease. Nature 2006; 444: 875-880.

38 Yoshida M, Tomiyama H, Yamada J, Matsumoto C, Odaira M, Shiina K et al. Relationship of insulin resistance to macro- and microvasculature reactivity in hypertension. Am J Hypert 2010; 23: 495-500.

39 Shimokawa H. Primary endothelial dysfunction: atherosclerosis. J Mol Cell Cardio 1999; 31: 23-37.

40 Cynober LA. Plasma amino acid levels with a note on membrane transport: characteristics, regulation, and metabolic significance. Nutrition 2002; 18: $761-766$.

41 Pollock JS, Förstermann U, Mitchell JA, Warner TD, Schmidt $\mathrm{HH}$, Nakane M et al. Purification and characterization of particulate endothelium-derived relaxing factor synthase from cultured and native bovine aortic endothelial cells. Proc Natl Acad Sci USA 1991; 88: 10480-10484.

42 Luscher TF, Vanhoutte PM. The Endothelium: Modulator of Cardiovascular Function. CRC Press Inc.: Boca Raton, FL, USA, 1990. 
43 Naseem KM. The role of nitric oxide in cardiovascular diseases. Mol Aspects Med 2005; 26: $33-65$.

44 Tanner FC, Meier P, Greutert H, Champion C, Nabel EG, Luscher TF. Nitric oxide modulates expression of cell cycle regulatory proteins: a cytostatic strategy for inhibition of human vascular smooth muscle cell proliferation. Circulation 2000; 101: $1982-1989$.

45 Tedgui A, Mallat Z. Cytokines in atherosclerosis: pathogenic and regulatory pathways. Physiol Rev 2006; 86: 515-581.

46 Braunersreuther V, Mach F, Steffens S. The specific role of chemokines in atherosclerosis. Thromb Haemost 2007; 97: 714-721.

47 Ross R. Atherosclerosis: an inflammatory disease. N Engl J Med 1999; 340 $115-126$.

48 Libby P, Ridker PM, Maseri A. Inflammation and atherosclerosis. Circulation 2002; 105: $1135-1143$.
49 Kim JA, Montagnani M, Koh KK, Quon MJ. Reciprocal relationships between insulin resistance and endothelial dysfunction: molecular and pathophysiological mechanisms. Circulation 2006; 113: $1888-1904$.

50 Wheatcroft SB, Williams IL, Shah AM, Kearney MT. Pathophysiological implications of insulin resistance on vascular endothelial function. Diabet Med 2003; 20: $255-268$.

51 Kearney MT, Duncan ER, Kahn M, Wheatcroft SB. Insulin resistance and endothelial cell dysfunction: studies in mammaliam models. Exp Physiol 2007 93: $158-163$.

(c) This work is licensed under the Creative Commons AttributionSOMERIIIHISRESERVED NonCommercial-No Derivative Works 3.0 Unported License. To view a copy of this license, visit http://creativecommons.org/licenses/by-nc-nd/3.0/

Supplementary Information accompanies the paper on International Journal of Obesity website (http://www.nature.com/ijo) 